\title{
Horizons/Théâtre
}

Revue d'études théâtrales

10-11 | 2017

Genre et arts vivants

\section{Post-pornographie et mélange de genres : l'exemple des Auto-porn box}

\section{Lisa Cogniaux}

\section{(2) OpenEdition}

Journals

Édition électronique

URL : http://journals.openedition.org/ht/532

DOI : $10.4000 /$ ht. 532

ISSN : 2678-5420

Éditeur

Presses universitaires de Bordeaux

\section{Édition imprimée}

Date de publication : 1 juillet 2017

Pagination : 136-150

ISSN : 2261-4591

\section{Référence électronique}

Lisa Cogniaux, « Post-pornographie et mélange de genres : l'exemple des Auto-porn box », Horizons/ Théâtre [En ligne], 10-11 | 2017, mis en ligne le 01 juillet 2018, consulté le 21 juillet 2019. URL : http:// journals.openedition.org/ht/532 ; DOI : 10.4000/ht.532

\section{(ब) $\Theta$}

La revue Horizons/Théâtre est mise à disposition selon les termes de la Licence Creative Commons Attribution - Pas d'Utilisation Commerciale - Pas de Modification 4.0 International. 


\section{LISA COGNIAUX}

Lisa Cogniaux est diplômée d'un Bachelier en Piano classique décerné par le Koninklijke Conservatorium van Brussel et d'un Master en Arts du spectacle vivant décerné par l'Université Libre de Bruxelles. Elle a rédigé un mémoire de recherche sur la question de la post-pornographie et de l'intimité, en se posant la question suivante : comment l'intimité peut-elle devenir un acte politique et militant? ("Post-pornographie et féminisme : vers une intimité publique? », mémoire sous la direction de Karel Vanhaesebrouck, ULB, 2015). Elle s'intéresse à l'activisme queer et aux points d'intersection entre format artistique et contenu politique, ainsi qu'à l'utilisation de la sexualité dans le spectacle vivant. Elle travaille actuellement en tant que dramaturge et assistante à la mise en scène dans le milieu du théâtre belge francophone (notamment pour le spectacle Do you wanna play with me?, KVS, janvier 2018).

Mail : cogniauxl@gmail.com

Résumé : La post-pornographie émerge dans le spectacle vivant en France dans les années 2010. Forme hybride, elle utilise le corps et la sexualités des performeu.r.se.s pour interroger les notions actuelles de genre et de sexualité. Dans cet article, l'autrice met en évidence le lien entre la forme artistique et le contenu politique des oeuvres : la disposition spatiale ainsi que l'interdisciplinarité présente dans la post-pornographie, et donc le mélange de genre artistiques, permettent en effet aux genres sociaux d'être déstabilisés. L'interaction entre image filmée et corps vivant, particulièrement, est mise en avant comme manière de déconstruire les acquis et de bousculer les attentes des spectateurices. Des performances déambulatoires de la compagnie à contre poil du sens, intitulées les Auto-porn box, sont utilisées comme exemple de ce phénomène. Ces auto-

Abstract: Angélica Liddell, a Spanish playwright and Post-porn appears in the living arts in France around 2000. This hybrid art uses the performer's bodies and sexualities to challenge the current notions about gender and sexuality.

In this paper, the author wants to emphasize on the connection between artistic form and content. The spatial arrangements and the interdisciplinarity presented in the postporn - the mixing of "genres » in the French signification of the word - question gender. Specifically, the interaction between the living body and the screen is an efficient way to deconstruct the expectations of the spectators and to question their own knowledge and experiment about gender or sex. In order to examine this phenomenon, the author chose itinerant performances called " auto-porn portraits sexuels ont pour point commun de partir de l'intimité sexuelle du performeurse pour remettre en question les codes de genres et de sexualités.

Les performances emmènent les spectateurices dans différents lieux, habituellement non dédiés au spectacle, brovillant ainsi les frontières entre espace de jeu et espace quotidien. De plus, dans chacune d'entre elles, plusieurs médias artistiques sont utilisés. L'article postule que cette interaction entre différents médias ainsi que le déplacement géographique des spectateurices inclut une agentivisation de celleux-ci, faisant de la post-pornographie un outil de réformement social et de remise en question efficace.

Mots-cLÉs : post-pornographie, agentivité, performances, performativité

box » by the company à contre poil du sens. These sexual self-portraits departing from the intimacy of the performer in order to question the sexual codes. This article describes and analyzes four of these performances. The performances take the spectators to different locations, some of which are usually not dedicated to art shows or performances. As a result the borders between extra and ordinary spaces are unclear. In each of the performances, differents medias are used. This interaction between medias and the geographic locations allow the spectators' increasing agency and turn the post-porn into an efficient tool for social reformation.

KEYWORDS: post-porn, agency, performativity, performance 


\section{Post-pornographie et mélange de genres : l'exemple des Auto-porn box}

La post-pornographie constitue un mouvement artistique hybride qui s'ancre dans les théories queers de la performativité ${ }^{1}$. Nommée et théorisée depuis peu ${ }^{2}$, il est difficile de la définir en raison de sa volonté de flouter les catégories déjà établies. Ainsi, même si la post-pornographie utilise toujours l'idée de la performance ${ }^{3}$, elle peut s'exprimer via différents médias ${ }^{4}$.

La post-pornographie est un courant artistique militant qui utilise la pornographie pour, entre autres, visibiliser des sexualités et des genres non normés. D’après Cha Prieur, « la prolifération des genres peut se comprendre comme un moyen de déstabilisation et comme une transition vers une autre réalité sociale ${ }^{5} \gg$, genre étant ici entendu dans une perspective queer. Je pense que la post-pornographie permet cette prolifération de genres, et que cette volonté a pour corollaire le dépassement des catégories artistiques. La postpornographie mélange donc les genres dans tous les sens du terme : en tant que catégories artistiques et en tant qu'identité performative et fluide.

En prenant l'exemple de quatre des performances Auto-porn box de la compagnie à contre poil du sens ${ }^{6}$, j'étudierai comment l'alternance de plusieurs médias et catégories artistiques - documentaire filmé, performance, arts plastiques - ainsi que la disposition spatiale éveillent l'agentivité des spectateurices et, par là même, permettent à la post-pornographie de devenir un outil militant efficace.

Pour ce faire, je reviendrai tout d'abord sur le concept même de postpornographie et présenterai ses ancrages théoriques. Ensuite, j’étudierai la démarche de la compagnie à contre poil du sens en décrivant les performances Auto-porn box.

\section{Notes préliminaires sur la post-pornographie}

\section{Origine et définition}

Rachele Borghi considère que la première performance pornographique date de 1990 avec The Public Cervix Announcement par Annie Sprinkle 7 . Cette 
performance consistait à montrer le col de l'utérus de l'artiste, porn-star, mais aussi docteure en sexologie et réalisatrice de films pornographiques. D’après Borghi, « The Public Cervix Announcement a définitivement marqué le passage d'une production porn mainstream ou grand public à un porn doté d'un contenu politique et d'objectifs de transformation sociale ${ }^{8} \gg$. En effet, au cours de cette performance, Annie Sprinkle dénonce entre autres le contrôle médical exercé sur le corps de la femme, se posant en tant que sujet de sa sexualité. En brisant les limites entre privé et public, elle met en place les bases d'une pornographie politique, faisant de sa propre sexualité mise en scène un outil féministe. Elle n'est pas la seule à viser cette transformation sociale grâce à la pornographie : depuis les années quatre-vingt-dix, des artistes féministes utilisent la pornographie de manière politique dans leurs œuvres, comme l'analyse Julie Lavigne dans sa thèse sur l'art féministe ${ }^{9}$. Mais le terme « post-pornographie » n'apparait comme désignant un courant artistique que dans les années $2000^{10}$. Néanmoins, il est difficile de définir exactement ce genre. En effet, suivant les pays et les artistes, le post-porn peut viser ou non des motifs excitatoires, plus ou moins violents ou explicites, intellectuels ou sensoriels, en bref il ne répond pas à des codes précis. De plus, la post-pornographie n'est pas cantonnée à un seul médium.

Ce qui réunit les différentes définitions possibles de la post-pornographie est l'utilisation d'actes sexuels plus ou moins explicites pour bousculer les définitions binaires de la sexualité et du genre, trop souvent présentées comme immuables et anhistoriques. Ainsi, il peut s'agir de montrer sur scène des actes considérés comme anormaux et cantonnés uniquement à la sphère privée, comme le fait Diana Pornoterrorista dans des performances où elle pratique le fist-fucking ${ }^{11}$, ou encore de remettre en question la nature même $\mathrm{du}$ sexuel, comme le font certaines vidéos du groupe Post $\mathrm{Op}^{12}$, toujours de « [représenter ou analyser] les représentations du "sexuel" hors des codes et des normes dominantes ${ }^{13} \gg$.

\section{Post-pornographie et performances studies}

Si la post-pornographie peut passer par différents médias artistiques et prendre des formes plus ou moins explicites, elle utilise toujours le performatif. J'utiliserai ici le terme « performatif » dans le sens où l'entend Judith Butler quand elle parle du genre ${ }^{14}$, c'est-à-dire comme une interaction entre l'identité des individus, performée à travers leurs actes, et la réalité sociale. Butler développe l'idée que le genre est une construction qui se développe à travers nos discours : ceux-ci sont influencés et s'inscrivent dans une réalité donnée, tout en la reformulant. Le genre est donc une performance sans cesse 
réitérée - en l'occurrence dans Trouble dans le genre, une performance de la masculinité ou de la féminité. C'est ce qui forme la performativité du genre.

Teresa De Lauretis rejoint Judith Butler lorsqu'elle évoque les technologies de genre ${ }^{15}$, qui sont d'après elle l'ensemble des institutions, discours et cultures qui produisent le genre. Dans cette perspective, la pratique artistique pourrait être envisagée comme technologie culturelle au service de l'idéologie dominante : les productions artistiques contribueraient à former ou à consolider une certaine réalité et un certain cadre. Comme un des cadres de notre société est l'hétéronormativité ${ }^{16}$, beaucoup d'œuvres artistiques reproduisent les genres et les sexualités comme binaires : masculin/féminin, homo/hétéro. Ce cadre est, tout comme le genre, réifié et naturalisé, c'est-àdire qu'il n'est pas perçu comme un cadre pouvant être modifié, mais qu'il semble découlé de l'ordre naturel des choses ${ }^{17}$. Le moyen de s'échapper de ces représentations passe par la subversion : il s'agit de modifier les actes dans le cadre préétabli de manière à révéler l'existence de ce cadre et par conséquent à pouvoir le modifier. Les sujets sont aussi agents, c'est-à-dire capables de modifier leur condition d'existence en prenant en compte leur ancrage performatif. C'est ce que fait la post-pornographie, en citant et pastichant un langage pornographique pour le détourner et le déconstruire.

En résumé et à la lumière de ces théories, la post-pornographie, en utilisant la pornographie, veut produire des corps qui font les genres au-delà de la binarité masculin/féminin et les sexualités, en dehors du cadre hétéronormé. Elle se situe dans un moment précis, entre cellui qui agit et celleux ${ }^{18}$ qui le regardent. Il ne s'agit pas de représenter ce qui est réel, mais de créer ensemble, entre artiste et public, ce moment de subversion. La subversion ne passe pas uniquement par le faire des performeurses, qui proposent une forme, mais bien par ce qui se passe entre ce faire et sa réception. La post-pornographie advient non pas en tant que forme, mais en tant que discours mouvant, qui défait les prescriptions d'identité ${ }^{19}$.

En conclusion, la post-pornographie met en pratique les performance studies dans un but de réaménagement social. Elle met en scène, comme le dit Annie Sprinkle : «better clichés, deconstructed clichés, performative clichés ${ }^{20} \gg$.

\section{L'exemple des Auto-porn box}

Les Auto-porn box sont un ensemble de six performances déambulatoires. Chacune est portée par un performeurse différentE. Les performeurses ont chacun.e conçu leur performance Auto-porn box avec pour thème leur propre sexualité. Elles constituent donc des autobiographies sexuelles, « un parcours 
d'auto-portraits documentaires et performatifs et un regard sur un univers et un parcours sexuel singulier ${ }^{21} \gg$.

Je les ai vues lors de leur création au festival Explicit, en mai 2015, au CDN de Montpellier. Les spectateurices étaient conduitEs de salle en salle pour assister aux différentes performances ; deux parcours différents étaient proposés dans trois salles. Une partie des spectateurices commençaient par la salle 1 , puis la 2 , puis la 3 , alors que l'autre partie faisait le trajet en sens inverse. Les performances étaient conçues pour recevoir soixante-dix personnes.

Je présenterai tout d'abord brièvement la compagnie et les performeurses du spectacle étudié, afin de contextualiser les œuvres et de mettre en évidence le lien entre militantisme et activité artistique. Ensuite, je décrirai quatre des six performances afin de mettre en avant le contenu artistique militant et politique de remise en question des binarités sexuelles. Je m'attarderai enfin sur le mélange de genres artistiques proposé dans les performances choisies, et particulièrement sur l'alternance entre écran et corps vivant.

\section{La compagnie à contre poil du sens et les performeurses des Auto-porn box}

La compagnie est fondée en 2005 à Montpellier par Matthieu Hocquemiller, alors jeune chorégraphe. Il s'intéresse à l'écriture plastique d'œuvres chorégraphiques : la vidéo, ainsi que les lumières, ont toujours été un médium important pour ses œuvres. Depuis quelques années, il considère les corps comme « enjeux politique et la danse comme un espace de connexion physique et de liens ${ }^{22} \gg$. Parallèlement à sa recherche artistique, il poursuit une recherche à l'EHESS dans le master « Genre, politique et sexualité ». Sa démarche se situe à l'intersection de l'esthétique du militantisme, et de la réflexion sur la place des sexualités.

Les performeurses des Auto-porn box font touTEs partie, d'une manière ou d'une autre, d'une minorité sexuelle. Qu'illes soient travailleurses du sexe, trans, queers, illes croisent leurs pratiques artistiques avec leur identité sexuelle et utilisent leur corps comme objet à la fois autobiographique et réflexif, puisqu'illes se prennent elleux-même comme sujet de leur proposition artistique. Le corps sexué et sexuel est alors remis en question afin d'interroger, à son tour, les corps des spectateurices, notamment grâce au dispositif spatial mis en place dans les performances comme je le développerai plus bas.

\section{Analyse des performances}

La première performance décrite a été créée et performée par Kay Garnellen, activiste trans et travailleur sexuel. 
Pour assister à celle-ci, les spectateurices sont conduitEs dans une salle en passant par les coulisses du théâtre. Une fois le public installé, le performeur apparaît alors qu'il était derrière un paravent situé à jardin. Il se place dos au public, en caleçon. Son caleçon est rembourré au niveau des fesses par deux ballons. Une table, sur laquelle sont posées des seringues et des boîtes de médicaments, est placée à côté de lui. Il s'enfonce les seringues, une à une, dans ses fausses fesses.

Un écran est placé en fond de scène sur lequel sont projetées des images d'une jeune butch $^{23}$. Petit à petit, il est possible de comprendre que cette fille est en fait Kay Garnellen. L'évolution de sa transition est résumée en images : la butch est de plus en plus masculine, jusqu'à la prise d'hormones qui stimule sa pilosité. Une photographie d'elle à l'hôpital avant et après sa mammectomie, des images de lui pendant le tournage de films pornographiques, défilent. Pendant la projection, Kay Garnellen continue à se piquer avec les seringues. Lorsqu' il les a toutes utilisées, les images s'arrêtent. Il se retourne, enlève son caleçon et dévoile ainsi son corps trans, celui d'un garçon avec une vulve.

La performance est autobiographique ; Kay Garnellen utilise des photos personnelles. En effet, le corps apparaît alors comme le résultat du processus de transition montré en images, ce qui le transqueerise ${ }^{24}$. Les images figées nous montrent l'évolution du corps du performeur : la transition de l'état « fille » avec toutes les caractéristiques physiques (poitrine, pas de pilosité faciale, etc.), à l'état « garçon » (pilosité, mammectomie, etc.). Mais Kay Garnellen rejoue sa transition par l'action symbolique de se piquer les fesses. La présence simultanée de deux médias permet d'envisager cette transition comme le passage d'un genre à un autre effectué grâce à la chirurgie et aux hormones, mais également comme la réitération performative d'une identité fluide et évolutive.

L'image finale présentée au public est celle d'un performeur garçon qui ne possède pas de pénis : il ne se conforme donc pas physiquement aux représentations habituelles du corps des hommes. Cependant, cette nonnormativité sexuée n'en fait pas un être dépourvu de sexualité : il est montré lors de tournages de films pornographiques ou de fêtes dans des clubs, avec des individus de différents genres, parfois en tant que pénétrant à l'aide d'un gode-ceinture, et parfois en tant que pénétré. L'idée selon laquelle sexualité et genre sont forcément liés est ainsi remise en question, puisque la sexualité de Kay Garnellen n'est pas liée à son identité de genre ; les binarités fille/garçon, pénétrant/pénétré, hétéro/homo sont mises à mal. 
La deuxième performance que je décrirai est celle de Marianne Chargois, militante au STRASS [Syndicat du Travail Sexuel], performeuse, travailleuse sexuelle en tant que dominatrice. Elle se déroule dans la même pièce.

La table sur laquelle sont posées les seringues est remplacée par une autre table sur laquelle se trouvent un ordinateur ouvert et une lampe de bureau. Marianne Chargois entre du même endroit que Kay. Elle est habillée avec un baggy, des boots noires et un sweat-shirt dont la capuche lui recouvre la tête, tenue unisexe qui rend sa silhouette androgyne. Elle s'assied sur une chaise, dos au public, face au bureau. Elle lit dans un micro des e-mails qu'elle a reçus, imprimés sur des feuilles de papier posées à côté de l'ordinateur. Ceux-ci contiennent les scénarios que ses clients lui soumettent avant de la rencontrer, dans lesquels ils explicitent comment ils veulent être humiliés et frappés. Des enregistrements de certaines séances passent, en alternance avec la lecture des e-mails. Il s'agit de dialogues entre certains clients et Marianne Chargois qui se fait appeler «Nurse Zoé ».

Après cette lecture, elle se lève et se déshabille : elle porte, en dessous de sa tenue de camouflage, un haut moulant, un string, des vêtements de playmate traditionnelle, et un gode-ceinture. Elle se met à danser comme pour un strip-tease tandis que des images sont projetées sur l'écran. Marianne Chargois y porte les mêmes vêtements que ceux qu'elle a sur scène. Dans une première séquence filmique, elle frotte le sol. Dans une seconde, elle sert de la nourriture sur un plateau. Les actions quotidiennes qu'elle effectue renvoient à une féminité domestique, mais cette image d'elle est vite troublée par la troisième séquence filmique dans laquelle elle lave des godes en grand nombre, qui renvoient à une sexualité contra-sexuelle ${ }^{25}$ qui n'a pas besoin de pénis pour être pénétrante. La présence de Marianne Chargois réunit ces apparentes contradictions : Cendrillon, une pute, une dominatrice, une fille qui saigne chaque mois, une fille qui vit sa sexualité en dehors de ce que la pornographie mainstream ${ }^{26}$ présente comme « vrai sexe », avec un pénis qui finit par éjaculer ${ }^{27}$. Marianne Chargois se place en tant qu'agent maître de son corps. Elle n'est pas victime et ses pratiques sont présentées de manière non moraliste et indépendante de son genre. Ceci contribue à bousculer les idées reçues concernant les rapports de pouvoir présents à la fois dans la division genrée, dans le travail sexuel et dans la pratique $\mathrm{BDSM}^{28}$.

Pour la troisième performance, celle de Ludovic Lézin, chorégraphe et danseur homosexuel, les spectateurices sont invitéEs à changer de salle et même de bâtiment. Conduits dans une cour à cinq minutes à pied du bâtiment 
initial, illes entrent dans une salle plongée dans le noir. Le public est invité à rester debout dans une salle dont les murs sont parés de trois écrans. Sur le premier, un gros plan du torse nu de Ludovic Lézin est projeté. Il présente une sorte de tutoriel sur la pratique de la baguette chinoise, accessoire qui pince les tétons utilisé dans les jeux BDSM, tandis qu'une musique techno est diffusée à bas volume. Le deuxième écran s'allume ensuite sur une capture d'écran d'un site internet d'escorts masculins destiné aux hommes homosexuels. La voix de Ludovic Lézin explique comment le site fonctionne. Ensuite, sur le troisième écran, Ludovic Lézin, dont la tête est toujours hors champ, explique cette fois-ci comment mettre une sonde urétrale, images à l'appui.

Enfin, les trois écrans s'allument en alternance, invitant les spectateurices à se tourner à chaque fois d'un côté différent. Le volume de la musique techno augmente, jusqu'à couvrir la voix de Ludovic Lézin enregistrée dans les vidéos. À ce moment-là, le performeur, qui était dans le public, se déshabille et commence à danser au milieu des spectateurices. Il rampe, se redresse et prend des poses, debout contre certaines personnes du public. Pour finir, la musique s'interrompt, les écrans s'éteignent et il passe derrière l'un d'eux. Le ton explicatif neutre des vidéos, à propos de pratiques sexuelles marginales, les inscrit dans un genre documentaire, mais tout comme dans la performance de Kay Garnellen, la présence corporelle de Ludovic contredit le caractère informatif : la théâtralité de son corps place le texte ${ }^{29}$ transmis par les images dans une présence subjective et en mouvement. Alors que le public était en dehors de l'histoire racontée, qui se déroulait sur écran, il se retrouve au centre de celle-ci. L'homme dont il est question est au centre de la pièce, dans l'espace qui semblait réservé aux spectateurices. Celleux-ci ont alors un choix à faire puisqu'illes doivent soit se déplacer pour éviter d'entrer en contact avec le corps nu de Ludovic, soit rester sur place et le toucher, voire gêner ses mouvements. Ces mouvements de foule créent une interaction avec le performeur, mais également entre spectateurices.

Qu'illes le veuillent ou non, les spectateurices deviennent également performeurses : l'espace du public et l'espace du performeur sont réunis. Leurs réactions influencent la spatialité et le déroulement du spectacle. La vidéo explique crûment une certaine réalité du travail sexuel et des pratiques BDSM, et le corps rappelle que cette réalité est subjective et unique, bousculant à la fois physiquement et mentalement les spectateurices. Illes passent de la passivité possible d'un public de cinéma - ce qu'il y a sur l'écran est déjà tourné, l'acteur et le réalisateur sont à priori absents, que l'on s'y intéresse ou pas ne change rien à la projection - à l'activité exigée par une performance 
qui les bouscule physiquement. Le mouvement et le changement de statut soudain amènent à changer notre regard sur le travail sexuel, les pratiques BDSM et l'homosexualité masculine.

Pour la performance suivante, les écrans ont été enlevés, des sièges sont installés. Le performeur est Mathieu Jedrazac. Une porte claque au fond de la salle et il arrive en courant, habillé d'une petite robe et de chaussures à talons hauts. Une musique pop éclate, et il se met à rapper : « Putain c'est quoi ça ? C'est du sperme. » Il enchaîne ainsi des chansons autobiographiques sur son parcours : la masturbation devant les films pornographiques hétérosexuels où il imaginait qu' il avait un vagin et non un pénis, le passage à l'acte et le comingout, la fréquentation d'hommes qui n'assumaient pas leur homosexualité. Durant les intermèdes entre les chansons, il singe les codes du concert pop : il salue le public d'un « bonsoir Montpellier » et adopte la gestuelle d'une diva. Au cours de la performance, il allume deux projecteurs sur les côtés de la scène, qui diffusent en alternance des lumières de plusieurs couleurs, et jette des ballons gonflables sur la scène. Il porte ensuite un gode qui représente de manière réaliste un pénis, tourne sur lui-même en avant-scène, et fait fonctionner une machine à fumée. Il en résulte une ambiance entre celle du karaoké populaire et du club de strip-tease lugubre.

Mathieu poursuit en interprétant de manière très théâtrale « pace, pace » de Verdi avec une voix de contre-ténor. Au moment le plus dramatique du chant, il se place en avant-scène, soulève sa robe sous laquelle il ne porte rien et s'auto-pénètre sur le gode. Il chante la partie la plus aiguë et la plus forte en enfonçant le gode plus profondément dans son anus et continue à chanter un certain temps ainsi. Il enlève le gode et se relève à la fin de l'aria.

Enfin, sur un slow, il propose aux spectateurices de venir sur scène et d'inviter la personne qui leur ferait envie, mais qu'illes n'osent pas aborder. Pendant que le public danse, il est sur le côté et observe. Il chante ensuite une dernière phrase : « encule-moi, encule-moi ».

La performance reprend les codes de la culture pop en les subvertissant; en effet, il remplace les refrains convenus par son expérience marginale. Il joue avec les codes de l'homosexuel efféminé puis soudain les rompt : notamment quand il raconte ses expériences sexuelles crument, quand il rappe ou encore quand il explique adorer avoir un pénis, mais fantasmer sur l'idée d'avoir un vagin. Tout au long de la performance, il dévoile la mise en scène et la création de son personnage : par exemple lorsqu'il allume les projecteurs lui-même ou la machine à fumée, il nous montre la construction du cadre de sa prestation. 
Il déconstruit ainsi son image au fur et à mesure qu'il la produit ; les ballons gonflables sur scène ou encore le gode qui tourne sur lui-même sont autant de manières de montrer qu'il s'agit d'un jeu, kitsch et parodique.

Le geste radical de s'auto-pénétrer avec une prothèse pendant qu'il chante de l'opéra est le climax de ce processus : la pop-star devient à cet instant à la fois porn-star et diva. Rachele Borghi souligne que la post-pornographie a comme caractéristique l' « effacement de la frontière entre la culture légitime (l'art) et les productions culturelles illégitimes (la pornographie) ${ }^{30} \gg$. C'est exactement ce que fait Mathieu. Il joue sur des genres très différents : le concert pop, la pornographie, l'opéra, le strip-tease, le cabaret, en nous montrant que tous sont à égalité, construits en direct par sa performance, qu'il est possible de passer de l'un à l'autre voire de tout faire en même temps. Son corps et sa voix ne sont plus ni masculin, ni féminin, car chaque fois qu'il s'inscrit dans un de ces registres il l'efface aussitôt. En conséquence, les structures homo/ hétéro, culture classique/culture populaire, garçon/fille sont brouillées.

\section{Du corps à l'écran, espaces de performativité}

Les six Auto-porn box ont en commun de remettre en question les binarités (homo/hétéro, masculin/féminin, documentaire/fiction, culture populaire/ culture élitiste, espace du public/espace des performeurses) en mélangeant des espaces habituellement distincts, et ce de plusieurs manières.

Ces espaces sont tout d'abord géographiques : les spectateurices sont invitéEs à se déplacer pour suivre le spectacle, et si dans certaines performances la limite scène/salle est claire, dans d'autres le public est placé dans l'espace de la scène, voir la scène investit son espace - c'est le cas dans l'auto-porn box de Ludovic Lézin. De plus, les endroits où les performances se déroulent ne sont pas destinés au théâtre : ce sont des salles de répétitions ou de conférences, sans les équipements standards des salles de spectacle. Ce choix n'est pas anodin dans le cadre du festival Explicit, alors que les autres spectacles programmés se déroulaient dans la grande salle du CDN.

Ensuite, toutes les performances mélangent les genres et les catégories artistiques : des images filmées de type documentaire sont insérées chez Kay Garnellen et Ludovic Lézin. Mathieu Jedrazac utilise les codes des concerts pop ou classique. Marianne Chargois se contorsionne, faisant référence aux arts du cirque, alors que des vidéos énigmatiques passent derrière elle.

Ainsi, la forme des performances décloisonne les séparations à la fois géographiques et artistiques. Dans le même temps, le propos des performances empêche les binarités sexuelles et genrées de se reproduire. En effet, comme il 
a été dit dans la description des performances, le corps nu de Kay Garnellen, les attitudes mi-masculines, mi-féminines de Mathieu Jedrazac ou encore les vêtements de Marianne Chargois, tantôt baggy, tantôt strip-tease interrogent les conceptions traditionnelles du genre et de la sexualité, en proposant des genreS et des sexualitéS fluides. Ce double décloisonnement, dans la forme et dans le fond, a pour effet d'inscrire le questionnement intellectuel dans le corps des spectateurices. Illes sont mis physiquement dans des situations qui sortent d'une position fixe et déterminée lorsque Ludovic Lézin émerge parmi le public pour danser nu ou lorsque les spectateurices dansent un slow et que Mathieu Jedrazac chante « encule-moi, encule-moi ». Leur statut est toujours liminal, entre performeurses et spectateurices, changeant aussi régulièrement que le statut de ce qu'illes regardent : un documentaire ? du théâtre ? une performance ? un cabaret ?

Si la post-pornographie permet de faire proliférer les genres et les sexualités, cela passe par l'agentivisation du ou de la spectateurice : c'est-à-dire par le fait de lui donner un pouvoir d'influence sur la performance en cours, en regard et à rebours de l'image déjà filmée et écrite.

Dans la performance de Marianne Chargois, les quatre écrans simultanés interrogent l'action en cours. Dans celle de Ludovic Lézin, les images, commentées en direct, sont parfois proches de la pornographie, notamment quand il se met une sonde urétrale avec un gros plan sur un pénis en érection. Même si le but apparent n'est pas masturbatoire, l'image d'un pénis qui se fait pénétrer peut éveiller l'excitation. Pour finir, dans celle de Kay Garnellen, l'évolution de sa transition n'est pas tout de suite explicitée. Dans les performances de Marianne Chargois et de Kay Garnellen, l'interaction entre corps et écran permet à la fois de comprendre le propos et empêche d'en faire un « tout » indissoluble. En effet, dans la performance de Kay Garnellen, l'action de se piquer les fesses renvoie à sa transition et explicite ainsi les images. Dans la performance de Marianne, l'écran se scinde en quatre parties alors qu'elle-même se contorsionne en regardant les spectateurices, autant de paradoxes pour une femme, travailleuse sexuelle et personne pratiquant le BDSM. Le gode-ceinture qu'elle porte renvoie à la production genrée des corps ${ }^{31}$ alors que les images font référence à son statut de travailleuse sexuelle et de femme. Dans les deux performances, l'écran montre une part autobiographique et intime des performeurses que complète le corps vivant dans des subjectivités momentanées qui échappent à la catégorisation. La performance de Ludovic Lézin, similairement, échappe aux catégorisations, 
sauf que l'intervention du corps, au lieu d'expliciter le propos des images, le complexifie au contraire : ce qui était « documentaire » et « objectif » apparaît soudainement comme l'expérience subjective d'un corps en particulier, qui bouscule le public au sens propre. Les impressions qu'il a été possible d'éprouver en regardant les films sont remises en question par l'irruption du corps nu et dansant. Si les films interrogent les spectateurices, il n'est pas possible d'y réagir directement : leur issue ne changera pas. Par contre, les corps en face sont palpables. Leurs actions sont uniques, même si répétées. J'y vois un parallèle avec les théories de Judith Butler : les conditions d'existence, définies d'avance, sont les images filmées, tandis que la marge d'agentivité des individus se retrouve dans le corps vivant. Les performances répondent alors à la question qu'elle pose dans Trouble dans le genre : $\ll[. .$. quel genre de performance accomplira et révélera la nature performative du genre lui-même de manière à déstabiliser les catégories naturalisées de l'identité et du désiri ${ }^{32}$ ? » L'irruption du corps vivant devant l'image change l'interprétation possible des images, révélant leur nature performative. La déstabilisation de ce qui semble acquis est alors mise en route, ne serait-ce qu'inconsciemment, parallèlement à la déconstruction des schémas sexuels en vigueur. Les espaces de performativité se créent non seulement entre performeurses et spectateurices, mais aussi entre les différents médias, genres artistiques et lieux. Notamment du fait de l'aller-retour entre corps et écran, les spectateurices, agents, sont dans l'impossibilité d'être fixéEs sur ce qui est en train de se passer et de catégoriser le spectacle. Illes réagissent à des conditions d'existence qui se modifient sans cesse, ne sont pas déterminées, et par conséquent peuvent être subverties par le regard qu'illes posent dessus.

\section{Pour conclure}

Les auto-porn boxes accompagnent les buts visés par la post-pornographie, militantisme et forme artistique étant intrinsèquement liés. Le mélange des genres sociaux et artistiques présents dans les performances est un élément essentiel à cette nouvelle forme hybride. L'application pratique des théories de la performativité passe ainsi par l'entre : ni ici, ni là-bas, mais dans l'instant. Le public est également placé entre, dans une position pas toujours confortable qui le pousse à être agent de l'action en cours. Cet entre passe également dans le cas étudié, par une forme hybride : entre plusieurs catégories artistiques, plusieurs médias, entre la culture populaire et la culture élitiste. De cette manière, les évidences et les binarités sont remises en question. En effet, si la forme artistique post-pornographe ne fait partie d'aucune catégorie préalablement définie, elle reflète le propos militant qui veut faire éclater les 
codes de genres et de sexualités existants. Les artistes, en cassant les schémas attendus, en brouillant les séparations entre scène et salle, en mélangeant arts vivants et images filmées, déstabilisent nos acquis, physiquement et intellectuellement.

\section{Notes}

1. D'après Marie-Hélène/Sam Bourcier : «La théorie queer met l'accent sur la critique des genres en faisant éclater le cadre binaire hommes/femmes qui s'était finalement réindexé sur la différence sexuelle avec le féminisme, en explorant plutôt la production des masculinités et des féminités, des masculinités sans hommes et des féminités sans femmes, par exemple. » La théorie queer s'appuie sur la performativité dans le sens où elle défend l'idée que c'est la répétition de performances qui créé la fiction des genres comme identités fixes. Cf. Bernard Andrieu, «Entretien avec Marie-Hélène Bourcier. ", Corps n ${ }^{\circ}$ 4, janvier 2008, p. 5-11.

2. Rachele Borghi, une des premières autrices françaises à avoir écrit sur le sujet estime que la naissance du terme " post-porn » comme fédérateur d'une nouvelle communauté artistique date du Maratona Post-porn organisé en 2003 par Paul B. Preciado. Voir Rachele Borghi, « Post-Porn », Rue Descartes n 79, 03/2013, p. 31.

3. Le terme " performance » est ici entendu dans le sens que Richard Schechner, entre autres, lui donne : la performance est l'évènement qui se produit entre spectateurices et artistes, dans un moment déterminé. Il s'exprime ainsi : " the uniqueness of an event does not depend on its materiality solely but also on its interactivity - and the interactivity is always in flux ». Cette performance contribue à créer une réalité, dans un moment précis. En dehors du cadre théâtral, la performance existe également : entre une personne qui agit et son environnement. Cf. Richard Schechner, "What is performance? ", Performance studies: An introduction, Routledge, Londres, New York, 2006.

4. Le terme médium est sujet à caution ; je l'utilise ici dans le sens d'un moyen de transmission de l'information; ainsi, le théâtre est un médium, comme la télévision, la radio, internet peuvent l'être. La post-pornographie utilise ces différents canaux pour s'exprimer, voire en mélange plusieurs. Pour aller plus loin sur le théâtre comme médium : Christophe Balme, "Theater and media ", The cambridge introduction to theater studies, Cambridge, Cambridge University Press, 2008, p. 195-208.

5. Cha Prieur, Des géographies queers au-delà des genres et des sexualités??, [en ligne], Espace Temps, Travaux, 20 avril 2015. Consulté le 27 juin 2017. URL : http://www.espacestemps.net/articles/des-geographies-queers-au-dela-des-genres-et-des-sexualites/\#_ $\mathrm{ftn}$

6. Cette compagnie, fondée par Matthieu Hocquemiller, a entre autres organisé le festival Explicit qui s'est déroulé en mai 2015 à Montpellier, et a produit deux spectacles qui peuvent être considérés comme post-pornographiques : (Nou) et Auto-porn box. Voir Cie à contresens du poil, Explicit : festival d'expression plurielle du sexuel, [en ligne], 2015. Consulté le 29 avril 2015. URL : http://festival-explicit.fr/wpexplicit/

7. Rachele Borghi, « Post-Porn », op. cit, p. 29. 
8. Ibid.

9. Julie Lavigne, «L'art féministe et la traversée de la pornographie : Érotisme et intersubjectivité chez Carolee Schneemann, Pipilotti Rist, Annie Sprinkle et Mariene Dumas ", Thèse de doctorat en histoire de l'art, sous la direction de Christine Ross, Montréal, Université McGill, décembre 2004.

10. Rachele Borghi, « Post-Porn » op. cit., p. 30.

11. Le fist-fucking pourrait être considéré comme une pratique post-porn centrale. En effet, le fist-fucking est centré sur l'anus, dont sont pourvus à la fois les biohommes et les biofemmes - terme emprunté à Paul B. Preciado. De plus, on pourrait avancer que le fist-fucking fait de la main et du bras un gode, selon la technologie "godotectonique » développée par Preciado. Cette godotectonique permet de dégénitaliser la sexualité et de sortir de l'idée du gode comme imitation du pénis pénétrant. Voir Paul B. Preciado, Manifeste contra-sexuel, éditions Balland, Paris, 2000, p. 53.

12. Post op, Campos de castilla, Post-op, Castille, 2015, $5 \mathrm{mn}$.

13. Cie à contre poil du sens, Explicit : festival d'expression plurielle du sexuel. Consulté le 29 avril 2015. URL : http://festival-explicit.fr/wpexplicit/

14. Judith Butler, Trouble dans le genre : le féminisme et la subversion de l'identité, Paris, La Découverte, 2005 [1990].

15. Le terme «technologie culturelle » fait référence à Teresa de Lauretis lorsqu'elle parle de « technologie de genre »: Teresa De Lauretis, Théorie queer et culture populaire : de Foucault à Cronenberg, trad. Marie-Hélène Bourcier, Paris, La Dispute, 2007 [1987].

16. Notes de Cynthia Kraus sur la traduction de Troubles dans le genre: «Ce terme désigne le système, asymétrique et binaire, de genre, qui tolère deux et seulement deux sexes, où le genre concorde parfaitement avec le sexe (au genre masculin le sexe mâle et au genre féminin le sexe femelle) et où l'hétérosexualité (reproductive) est obligatoire, en tout cas désirable et convenable. ", Judith Butler, Trouble dans le genre : le féminisme et la subversion de l'identité, op. cit., p. 24.

17. Butler parle de fiction régularisée [de l'identité] lorsqu'on considère que l'homme et la femme préexistent comme des substances durables. Voir Ibid., p. 95.

18. J'utilise dans cette grammaire une grammaire dite inclusive : au lieu de considérer que le masculin vaut comme neutre en français, je fusionne les formes masculines et féminines. Exemple : ceux et celles devient celleux, spectateurs et spectatrices devient spectateurices, performeur et performeuses devient performeurses, etc. Cela me semblait essentiel d'utiliser une telle forme d'écriture dans un travail qui parle de la performativité, sachant que le langage est performatif. Cette structure (spectateurices au lieu de spectateur.trice.s) permet de créer des formes qui peuvent également être utilisées dans le langage parlé. Elle ne fonctionne pas toujours (notamment dans le cas d'adjectifs tels que différents et différentes, tous et toutes) et dans ce cas-là j’utilise une structure avec des majuscules qui indiquent le féminin : touTEs, différentEs, etc.

19. "Une généalogie critique a besoin d'être complétée par une politique des actes performatifs de genre, qui propose à la fois une redescription des identités genrées existantes et une prescription sur ce qui est souhaitable en matière de réalité de genre ", Judith Butler, Actes performatifs et constitution des genres : phénoménologie et théorie féministe, [1990], Outre-scène n 9 9, Strasbourg, mai 2007. 
20. Introduction par Gabrielle Cody dans Annie Sprinkle, Harcore from the Heart. The Pleasures, Profits and Politics of Sex in Performance, New York, Bloomsbury, 2001, p. 2.

21. Cie à contre poil du sens, Mattbieu Hocquemiller/Cie à contre poil du sens. Consulté le 11 mars 2016. URL : http://festival-explicit.fr/wpexplicit/

22. Ibid.

23. Femme qui assume une identité lesbienne masculine.

24. Terme utilisé par Bernard Andrieu, qui signifie l'acte militant de non seulement transformer son corps mais par-là même de le réinventer au-delà des binarités homme/ femme. Voir Bernard Andrieu, « La mise en scène de l'orgasme sur les scènes post-pornographiques ", conférence au colloque Érotisme et ethnoscénologie : l'érotisme et la sexualité dans les arts du spectacle, MSH Paris Nord, 26 février 2015. Consulté le 27 avril 2017. URL : https://www.youtube.com/watch?v=_H00IeCvHJI

25. Paul B. Préciado, Manifeste centra-sexuel, op. cit.

26. Le terme mainstream est réfuté par Florian Vörös dans son anthologie des porn studies parce qu'il conduit à une impasse intellectuelle qui veut que ce qui est consommé par le plus grand nombre « [reproduise] nécessairement les normes de représentations et les rapports de vision dominants ». Florian Vörös (dir.), Cultures pornographiques : anthologie des porn studies, Amsterdam, 2015, p. 20. Je le garderai malgré tout ici parce que j'utilise un article de Heather Butler, publié en français dans l'anthologie, mais que j'ai lu aussi en anglais avant sa traduction. Les termes anglophones mainstream pornography y sont entendus comme pornographie hétérosexuelle se pliant aux schémas habituels : préliminaires, pénétration et money shot c'est-à-dire éjaculation du pénis comme climax. Voir Heather Butler, «What do you call a lesbian with long fingers?: the development of lesbian and dyke Pornography ", dans Linda Williams (ed.), Porn Studies, Londres, Duke University Press, 2004.

27. Heather Butler, «Que dit-on d'une lesbienne aux doigts longs ? : le développement de la pornographie lesbienne et gouine ", dans Florian Vörös (dir.), Cultures pornographiques : anthologie des porn studies, op. cit., p. 169.

28. En effet, d'après Virginie Despentes, la vision majoritaire de la prostitution est celle d'une activité dégradante, et celles qui la pratiquent sont alors vues comme des victimes. Les pratiques BDSM, quant à elles, sont placées en bas de la hiérarchie des pratiques sexuelles selon Gayle Rubin. Cf. Gayle Rubin, « Le péril cuir : remarques sur la politique et le SM ", dans Gayle Rubin, Surveiller et jouir, antbropologie politique du sexe, [1975-2000, textes édités par Rostom Mesli], trad. Flora Bolter, Christophe Broqua, Nicole Claude-Mathieu et Rostom Mesli et alii, Paris, Epel, 2010, passim.

29. Texte au sens large du terme.

30. Rachele Borghi, «Post-Porn» op. cit., p. 29.

31. Preciado, Paul, Manifeste contra-sexuel, op. cit., p. 61.

32. Judith Butler, Trouble dans le genre: le féminisme et la subvention de l'identité, op. cit., p. 263. 